\title{
A MEMS ELECTROSTATIC PARTICLE TRANSPORTATION SYSTEM
}

\author{
A. Desai, S.W. Lee, and Y.C. Tai \\ Caltech Micromachining Laboratory, \\ Electrical Engineering 136-93, \\ California Institute of Technology, \\ Pasadena, CA 91125 USA
}

\begin{abstract}
We demonstrate here an electrostatic MEMS system capable of transporting particles $5-10 \mu \mathrm{m}$ in diameter in air. This system consists of 3-phase electrode arrays covered by insulators (Figs. 1,2). Extensive testing of this system has been done using a variety of insulation materials (silicon nitride, photoresist, and Teflon), thickness $(0-12 \mu \mathrm{m})$, particle sizes $(1-10 \mu \mathrm{m})$, particle materials (metal, glass, polystyrene, spores, etc), waveforms, frequencies, and voltages. Although previous literature [1-2] claimed it impractical to electrostatically transport particles with sizes 5-10 $\mu \mathrm{m}$ due to complex surface forces, this effort actually shows it feasible (as high as $90 \%$ efficiency) with the optimal combination of insulation thickness, electrode geometry, and insulation material. Moreover, we suggest a qualitative theory for our particle transportation system which is consistent with our data and finite-element electrostatic simulations.
\end{abstract}

\section{INTRODUCTION}

The ability to transport and manipulate particles in air is desirable in many instruments such as airborne particle samplers, particle sorters, and electrostatic particlecleaning apparatuses. There are many different ways to transport particles larger than $10 \mu \mathrm{m}$ such as forced air jets, centrifuges and other mechanical means. However for particles ranging from 1 to $10 \mu \mathrm{m}$, there is still no efficient way to transport them because complex surface forces, instead of gravitational force, dominate. This attribute has long been a bottleneck for the development of automated airborne pollutant samplers for biological spores, dust particles, and chemical agents because their sizes fall into the $1-10 \mu \mathrm{m}$ range.

In the past, successful transportation of $s u b 10 \mu \mathrm{m}$ particles has been accomplished in the liquid medium, but not on a solid surface in air. In air, Moesner and Higuchi [3] and Balachandran et al [4] have demonstrated motion of larger particles with voltages up to a few $\mathrm{kV}$. Unfortunately, in the sub $10 \mu \mathrm{m}$ regime the electrostatic forces that can be exerted on the particle are of the same magnitude as the adhesive forces between the particle and the solid surface. Numerous researchers have noted this size range cutoff; for example, Novick et al [2] have noted that the sub $10 \mu \mathrm{m}$ range presents a different regime where surface adhesive forces and particle charging hinder the successful engineering of a robust particle transport system.

The goal of this work then, is to reexamine this difficult task and design a dielectrophoretic (DEP) particle transportation system [3-5] capable of moving particles below $10 \mu \mathrm{m}$ in air with low voltages. Our work emphasizes a three-phase electrode array with specific insulative films. It is found that these films enable us to not only repeatably demonstrate motion for 5 and $8 \mathrm{um}$ particles, but reveal a novel phenomenon - the strong dependence of particle transportation efficiency with film thickness. In addition, computer simulation of DEP force on the particle is consistent with our observations and hypothesis.

\section{FABRICATION}

The particle transportation chip was developed in using standard microfabrication processes. The process basically consists of depositing two insulation layers and two conductive layers for a three-phase network of electrodes. A cross-sectional view of a finished electrode panel is shown below. (Fig. 1)

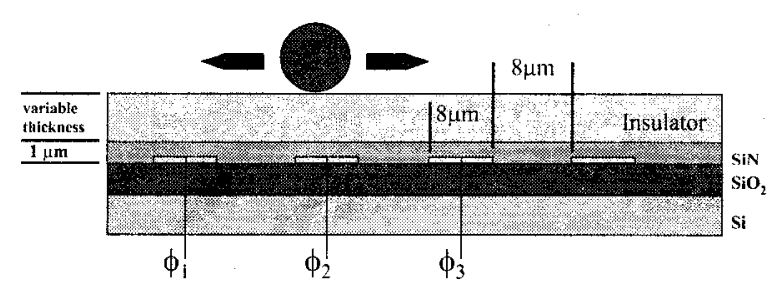

Fig. 1: Electrode cross-section

The fabrication sequence begins with a silicon substrate coated with 1-2 $\mu \mathrm{m}$ of thermal oxide. Next, $3000 \AA$ of aluminum is thermally evaporated and patterned to form the electrodes. Plasma-enhanced chemical vapor deposition (PECVD) silicon nitride is deposited to form 
a $1 \mu \mathrm{m}$ insulation layer between the $1^{\text {st }}$ and $2^{\text {nd }}$ metal layers. Vias are etched into this silicon nitride layer for connection of the $2^{\text {nd }}$ Al layer to the $1^{\text {st }}$. Next, the $2^{\text {nd }}$ Al layer is deposited and patterned. In addition to the above process a similar process using gold instead of aluminum and low temperature thermal oxide instead of PECVD silicon nitride was also developed. Finally, another insulation coating ranging from $1 \mu \mathrm{m}$ to $5 \mu \mathrm{m}$ is deposited to change particle adhesion characteristics. Details on the insulation material (photoresist and Teflon) will be discussed in the experimental section. Cross-sectional views of the process steps are shown below.

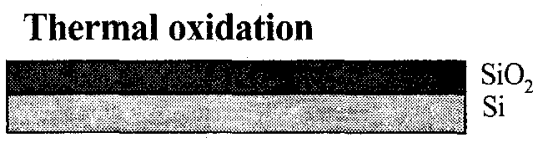

Insulator deposition \& patterning

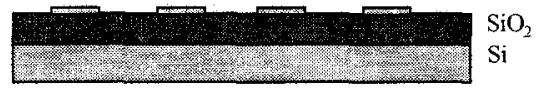

1st metal deposition \& patterning

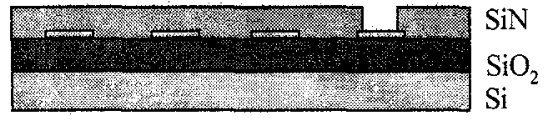

2nd metal deposition \& patterning

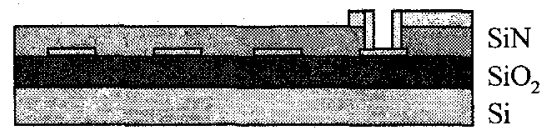

Final insulation coat

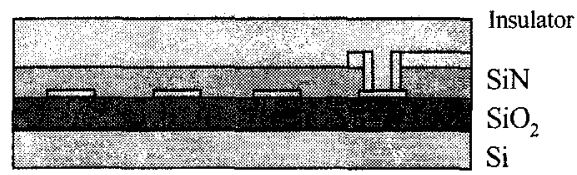

Fig. 2: Process flow for electrode chip

With the above process, various electrode designs were fabricated. Pictures below are examples of radial and linear geometries that were designed.

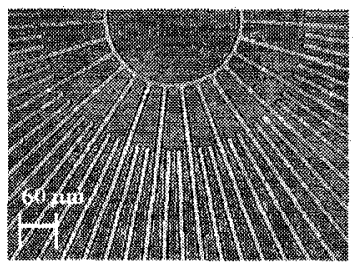

radial

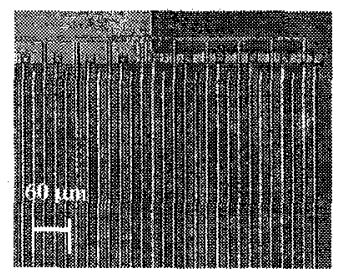

linear
Fig. 3: Various electrode structures

The electrode structures were operated with 3-phase circuit capable of waveforms from typically 20 to $200 \mathrm{~V}_{\mathrm{pp}}$ with frequencies from $0.1 \mathrm{~Hz}$ to $10 \mathrm{kHz}$. An example of the voltage patterns required for each phase is illustrated in the schematic below. (Fig. 4)

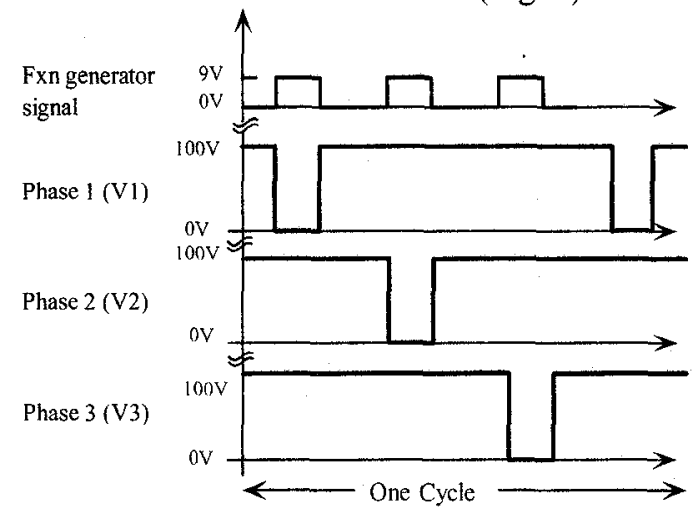

Fig. 3: Typical applied waveform

Other voltage patterns such as sinusoidal waves, triangular waves, bi-polar traveling waves, and the inverse of the waveforms shown in Fig.4 were also utilized for sustained particle motion. Particles can be made to change direction by switching phases 1 and 3 , and step (alternate) from one electrode to another by turning off one of the phases.

\section{PARTICLE FORCES}

\section{Dielectrophoretic Force}

Much work has been done in investigating the DEP force on particles ranging from dust to biological cells. The equation, from Jones[5], for the special case for a lossless dielectric sphere is

$$
F_{D E P}=2 \pi \varepsilon_{1} R^{3} K \nabla E_{0}^{2}
$$

The DEP force is a function of $\varepsilon_{1}$, the permittivity of the surrounding medium, $R$, particle radius, and $E_{0}$, the electric field. The constant $K$ is defined as

$$
K=\frac{\varepsilon_{2}-\varepsilon_{1}}{\varepsilon_{2}+\varepsilon_{1}}
$$

where $\varepsilon_{2}$ is the permittivity of the sphere. The important factor to note is that the DEP force is a strong function of the particle volume $\left(R^{3}\right)$ and the gradient of the electric field squared $\left(\nabla E_{0}{ }^{2}\right)$. It is the latter term that makes Eq. 1 difficult to compute in closed form for spherical particles.

\section{Adhesion Force}

The adhesion forces between a $\mu \mathrm{m}$-sized particle and a surface are mostly Van der Waals forces, electrostatic forces (image force), meniscus forces, and also gravitational forces.[7] It is impossible to calculate the exact contribution of these various forces that govern the adhesion of particles in this size range. However, the calculation of these forces on micro-particles, even 
in vacuum is approximate in order of magnitude at best.[6] In air, these theoretical formulas become more inaccurate because of irregularities of surfaces, molecular adsorption, and stray electronic charging. Nevertheless, we use these formulas to gain an understanding of the magnitude of the forces involved Many scientists have experimentally studied these phenomena, and have come up with estimates for adhesive forces.[7] Because of the irregularity of particle and surface contact areas, experimental results for this range of particles are often denoted in percentages of particles that are removed vs. adhered. Large variation is common with these percentages due to the statistical nature of the phenomena.

\section{EXPERIMENTAL}

A variety of particle sizes and materials were tested with this particle transportation chip. Most of the experiments were performed on linear electrode and spacing widths of $5 \mu \mathrm{m}$ and $8 \mu \mathrm{m}$ (pitch and width spacing is identical). Table 1 summarizes the types of particles tested.

Table 1: Particles tested

\begin{tabular}{|l|l|l|}
\hline Microspheres & $\varepsilon$ & $\begin{array}{l}\text { Mean } \\
\text { diameter }(\mu \mathrm{m})\end{array}$ \\
\hline Borosilicate glass & 5.8 & $5,8,11,15.4$ \\
Polystyrene DVB & 2.5 & $5,8.7$ \\
Paper Mulberry Pollen $^{*}$ & - & $11.1-15.6$ \\
Bermuda Grass Smut Spores" & - & $5.8-8.2$ \\
\hline \multicolumn{2}{|l}{ "refers to hydrated size }
\end{tabular}

Testing was conducted in the following fashion. First, the electrode panel was "turned on" and then particles were released on the electrode panel with the aid of a micromanipulator probe tip. As the probe tip neared the electrode panel surface, the traveling electric field force was sufficient to detach particles from the probe tip onto the surface of the transport chip. Multiple sets of experiments for each parameter (particle type, size, insulation, electrode spacing) were performed in this fashion. Particle efficiency data was gathered by noting the ratio of particles transported to the edge of the linear electrode array vs. the total number of particles on the structure. The laboratory environment was standard room temperature, $22^{\circ} \mathrm{C}$ and $30-60 \%$ relative humidity.

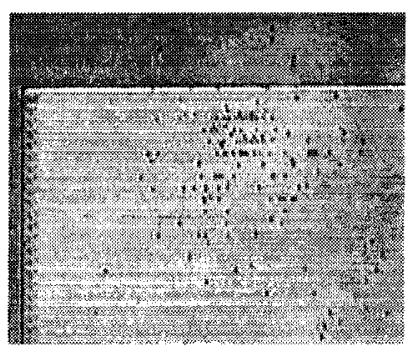

Fig. 5: Particles before applying waveform

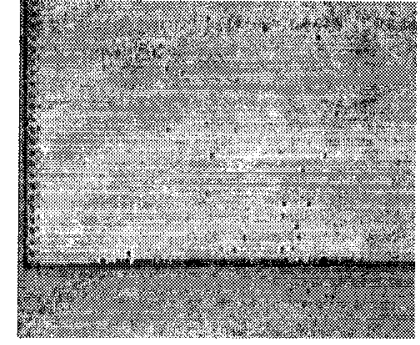

Fig. 6: Particle transported to edge of electrodes

\section{OBSERVATIONS}

Two major trends were noted during testing. Regardless of the type of final insulation films and their varying thicknesses, the transportation efficiency increased with increasing voltage. Secondly, the type of final surface coating also had a significant effect on particle efficiency and threshold voltage, the minimum voltage level to exhibit particle motion. Experiments using silicon nitride as the insulation layer resulted in poor performance. Large threshold voltages were needed, and moreover, particles would exhibit motion for few seconds and then stop moving. Motion would resume only when the voltage level was increased further. This was observed for voltage levels as high as $300 \mathrm{~V}$. Any further increase in voltage larger than $300 \mathrm{~V}$ resulted in breakdown of the insulation layer between the $1^{\text {st }}$ and $2^{\text {nd }}$ metal layers on the chip. In comparing different insulation materials, the effect was remarkably different. For example, photoresist film (hardbaked AZ 4400 ) resulted in maximum efficiencies for glass particles of approximately $45 \%$, but Teflon films[8] resulted in efficiencies of $80 \%$. Table 2 summarizes the behavior of different surface coatings.

Table 2: Linear $8 \mu \mathrm{m}$ spacing structure, $8 \mu \mathrm{m}$ glass particles

\begin{tabular}{|l|l|l|l|}
\hline $\begin{array}{l}\text { Surface } \\
\text { coating }\end{array}$ & $\begin{array}{l}\text { Frequency } \\
\text { range (Hz) }\end{array}$ & $\begin{array}{l}\text { Max } \\
\text { Efficiency }\end{array}$ & $\begin{array}{l}\text { Threshold } \\
\text { voltage } \\
\text { (V) }\end{array}$ \\
\hline $\begin{array}{l}\text { PECVD } \\
\text { silicon } \\
\text { nitride }\end{array}$ & $0.9-10$ & $10 \%$ & 80 \\
AZ 4400 \\
$\begin{array}{l}\text { Teflon (AF } \\
\text { 1601S) }\end{array}$ & $0.1-1000$ & $45 \%$ & 50 \\
\hline
\end{tabular}

Note: data has $+/-30 \%$ variation

In order to ascertain which adhesive forces (meniscus vs. Van der Waal vs. coulombic) contribute most to particle stiction, various surface modification treatments were performed. For example, the silicon nitride insulation was annealed at $400^{\circ} \mathrm{C}$ for $3 \mathrm{hrs}$ in order to remove surface moisture, and also, the chip was heated up to $200 \mathrm{C}$ while the particles were transported. The nitride surface was also silanized to 
increase surface hydrophobicity with a coating of trimethylcholorsilane (Sigma Corp.). However, the above treatments did not result in any significant improvement. Increase in surface roughness has also been known to reduce particle adhesion.[6] Consequently, the resist surface was roughened with $\mathrm{O}_{2}$ plasma which changed the characteristics of the motion. All the particles did not move in the same direction of the traveling wave, but rather, in a random fashion. This effect was probably due to the large amount of negative charge implanted on the resist surface from the plasma. Since no significant improvement was observed with the previous treatments, it is concluded that meniscus forces are not dominant factors in contributing to particle stiction

\begin{tabular}{|c|c|c|}
\hline $\begin{array}{l}\text { Surface } \\
\text { Treatment }\end{array}$ & $\begin{array}{l}\text { Contact } \\
\text { Potential }\end{array}$ & Harmonic Effect \\
\hline $\begin{array}{l}\text { Substrate heating } \\
\text { Annealing at } 400^{\circ} \mathrm{C} \\
\text { Plasma roughening } \\
\text { TMCS coating }\end{array}$ & $\begin{array}{l}\mathrm{Si}_{x} \mathrm{~N}_{y} \text { to glass } \\
\text { LTO to glass }\end{array}$ & $\begin{array}{l}\text { Full sinusoidal wave } \\
\text { Bipolar wave } \\
\text { Positive voltage wave } \\
\text { Ground voltage wave }\end{array}$ \\
\hline
\end{tabular}

Table 3: Surface and waveform tests

As mentioned previously, on silicon nitride, particles would only exhibit momentary motion when voltage level was constantly increased. This type of phenomenon is most likely associated with particle to surface contact charging. However, some sources also point out that the difference in contact potentials between the particle material and the substrate material can causing particle charging during detachment.[7,9] Therefore, to investigate if this type of charging was occurring, we also fabricated electrode panels with low temperature oxide (LTO) instead of $\mathrm{Si}_{\mathrm{x}} \mathrm{N}_{\mathrm{y}}$. Nevertheless from our measurement data, we did not find any noticeable difference between LTO and nitride for glass particles. Different waveforms such as the inverse of the waveform in Fig. 4, bipolar, and triangular were also investigated to ensure particle motion was due to DEP force and not just particle charging. When the traveling wave in Fig. 4 was utilized, the particles traveled in the same direction as the waveform, and when this same waveforms was inverted (i.e. positive $100 \mathrm{~V}$ traveling pulse) the particle also moved in the same direction. From this observation, we can conclude that particle motion is dielectrophoretic and not due to charging.

However, the most significant and remarkable effect on performance was noted when insulation thickness was increased. Transport efficiency variation also is a strong function of final insulation film thickness. Fig. 7 shows the variation of transportation efficiency vs. insulation height for $8 \mu \mathrm{m}$ glass spheres for photoresist and Teflon.

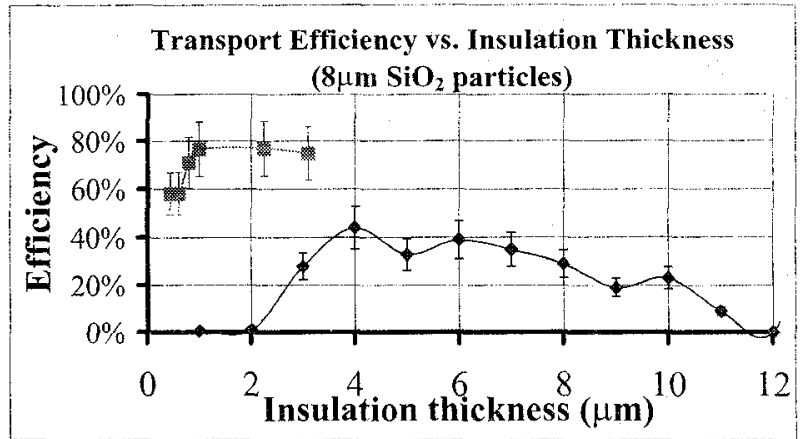

Fig. 7: Optimal thickness effect

In addition, Fig. 8 also shows the effect of frequency. As frequency increases the efficiency improves, and then saturates. For photoresist, motion cuts off at $\sim 1 \mathrm{kHz}$, and for Teflon motion was observed only up to $20 \mathrm{kHz}$ due to limitation of our circuit. This frequency window can be explained due to the dynamics of particle movement. There is a finite time associated with particle stepping from one electrode to another. This time involves particle acceleration and deceleration from one electrode to the next. At the cutoff frequency, the particle is unable to keep up with the traveling wave.

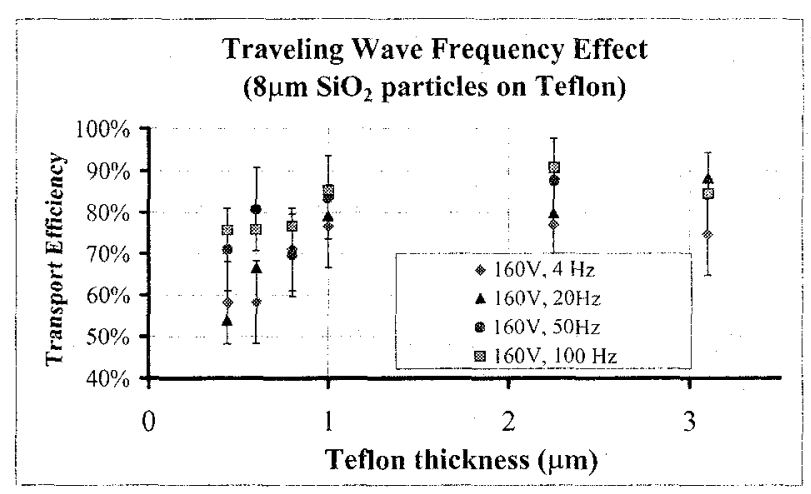

Fig. 8: Frequency effect on efficiency

\section{DISCUSSION}

Understanding the interaction between the DEP forces and adhesive forces on the particle is the key to interpretation of the experimental observations. From the experimental data, it seems quite possible that the image force contribution is the dominant adhesive force and decreasing this image force, $\mathrm{F}^{\mathrm{I}}$, leads to higher transportation efficiency. The image force arises when particles are charged and are in close proximity to a conductor.[5] In fact, the presence of an optimal thickness with the insulative films supports this hypothesis because $F^{1}$ is reduced when the distance from the particle to the electrodes is increased. 


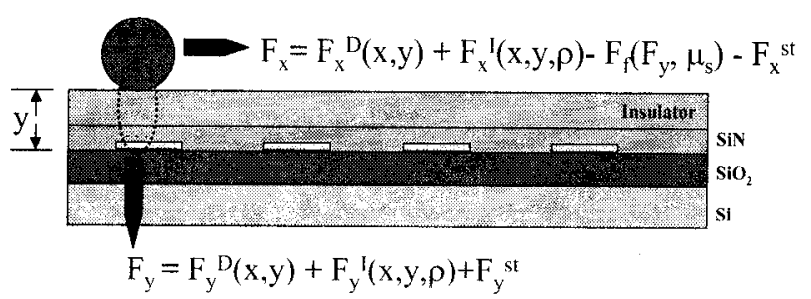

$\mathrm{F}_{\mathrm{D}}(\mathrm{x}, \mathrm{y})$ : driving force from applied voltage $\mathrm{F}_{\mathrm{I}}(\mathrm{x}, \mathrm{y}, \rho)$ : image force due charges on particle $F_{f}(x, y): \quad$ friction force, $\left\{\mu_{s} \bullet F_{y}(x, y)\right\}$

$\mathrm{F}_{\mathrm{st}}: \quad$ stiction forces

Fig. 9: Schematic of particle forces

Fig. [9] illustrates the various forces acting on the particle. The downward force on the particle, $F_{Y}$, arises from three sources: the image force, $\mathrm{F}_{\mathrm{y}}{ }^{ }$, the stiction force, $\mathrm{F}_{\mathrm{y}}{ }_{\mathrm{st}}$, and also the $\mathrm{y}$-component of the DEP force, $F_{y}{ }^{D}$. The horizontal force, $F_{x}$, can be separated into four parts: 1) the DEP force, $F_{x}{ }^{D}$, 2) $F_{x}{ }^{I}$, the $x$ component of the image force, 3 ) the frictional force, $F_{f}$, and 4) $F_{x}{ }^{\text {st }}$. However, $F_{f}$ is inherently related to $F_{Y}$ through the frictional coefficient, $\mu_{\mathrm{s}}$. Because of this relationship, any change in insulation thickness also has an effect on the net horizontal force on the particle.

To verify this downward component of the DEP force, $\mathrm{F}_{\mathrm{x}}{ }^{\mathrm{D}}$ and $\mathrm{F}_{\mathrm{y}}{ }^{\mathrm{D}}$ on a single particle have been computed using Maxwell, a 3-D finite element simulation.. (Fig.[10]) The simulation parameters are the following: $8 \mu \mathrm{m} \mathrm{SiO}{ }_{2}$ particle, $8 \mu \mathrm{m}$ electrode width and spacing, $100 \mathrm{~V}$ wave, particle distance from electrode is $3 \mu \mathrm{m}$, and the particle is uncharged. The electrodes and a particle are drawn to scale on the $\mathrm{x}$-axis. The $\mathrm{x}$ coordinate of each data point represents the position of the particle center with respect to the electrodes, and the $y$-axis shows magnitude of the force. From Fig. 9, we see that although the horizontal or $\mathrm{x}$-component of the DEP force, $F_{x}{ }^{D}$, due to the voltage waveform is responsible for particle transport, the DEP $y$ component, $F_{y}{ }^{D}$, acts downward thus adding to the frictional force. In other words, the DEP force not only exerts a horizontal force but a downward force as well.

DEP Force simulation for $8 \mu \mathrm{m} \mathrm{SiO}_{2}$ Particle

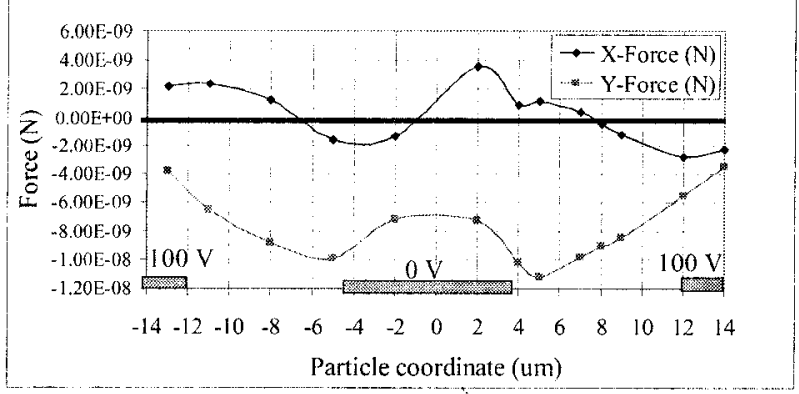

Fig. 10: DEP force on particle
Likewise in agreement with electrostatic theory, other simulation results show that $\mathrm{F}_{\mathrm{y}}{ }^{\prime}$ diminishes as the height from the electrodes is increased, and it is greatest when the particle is on top of the electrode. Superposition of these forces, image, DEP, and stiction gives us total downward force.

The FEM results corroborate the phenomena observed in experiment. In our experiments variation of this vertical distance which is controlled by the thickness of the insulation film, clearly affects the performance. If the insulation is too thin, the downward forces dominate and efficiency drops. If the insulation film is too thick the driving force is not enough to overcome the adhesive forces. Therefore at a specific thickness, $F_{X}$ is maximized and thus gives the highest transportation efficiency. However, each insulation film has its own optimal thickness (Fig. 7) because Van der Waals, meniscus force, and frictional coefficient between the particle and the insulation surface differ from film to film.

\section{CONCLUSION}

We have shown that particles of 5-10 $\mu \mathrm{m}$ size range can be manipulated electrostatically with voltages below $200 \mathrm{~V}$ in a standard room environment. In contrast to the work done in the past in this field, our work shows that it is possible to move $\mu \mathrm{m}$-sized particles in air. Furthermore, we have shown that particle charging is a significant cause of adhesion on our MEMS transportation system, and we have discovered a simple way to reduce its negative effect on particle transportation performance. Our goal is to incorporate this MEMS transportation system in an automated particle sampler.

\section{ACKNOWLEDGEMENTS}

The funding for this project was provided by DARPA, MicroFlumes Contract. N66001-96-C-83632. The authors would also like to thank Mr. X.Q. Wang and Mr. W. Hsieh for their knowledge and assistance with parylene and Teflon films.

\section{REFERENCES}

[1.] D.W. Cooper, H.L. Wolfe, J.T.C. Yeh, R.J. Miller, "Surface Cleaning by Electrostatic Removal of Particles," Aerosol Science and Technology, Vol. 13, 116-123, 1990.

[2.] V.J. Novick, C.R. Hummer, P.F. Dunn, "Minimum dc electric field requirements for removing powder layers from a conductive surface," Journal of Applied Physics, Vol. 65, 3242$3247,1989$. 
[3.] F.M. Moesner, T. Higuchi, "Devices for Particle Handling by an AC Electric Field," IEEE Conference Proc. MEMS 1995.

[4.] W. Machowski, W. Balachandran, SMIEEE, D. $\mathrm{Hu}$, "Influence of Electrode Geometry on Transport and Separation Efficiency of Powders Using Travelling Wave Field Techniques," IEEE, 1995.

[5.] T.B. Jones, "Electromechanics of Particles," Cambridge University Press, New York, 1995.

[6.] C.N. Davies, "Aerosol Science," Academic Press, London and New York, 1966.
[7.] A.D. Zimon, "Adhesion of Dust and Powder," Consultants Bureau, New York, 1982.

[8.] T. Hsu, W.H. Hsieh, K.Furutani, Y.C. Tai, “A Thin Film Teflon Electret Technology for Microphone Applications." Hilton Head 1996 Solid-State Sensor \& Actuator Workshop.

[9.] G.M.Colver, "Dynamic and stationary charging of heavy metallic and dielectric particles against a conducting wall in the presence of a dc applied electric field," Journal of Applied Physics, Vol. 47, No. 11, November 1976. 